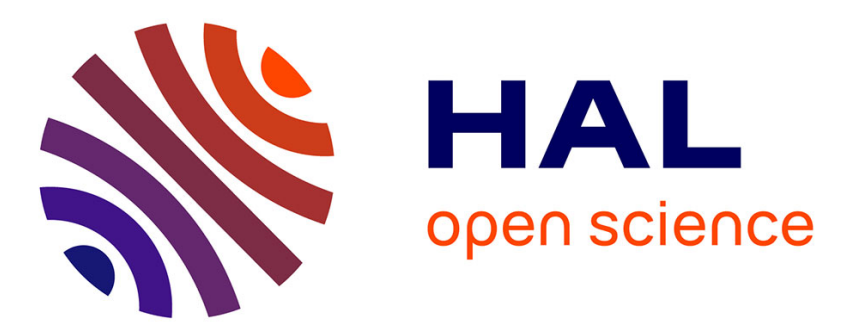

\title{
Considérations relatives aux représentations des substances corporelles en Nouvelle-Guinée
}

\author{
Pascale Bonnemère
}

\section{To cite this version:}

Pascale Bonnemère. Considérations relatives aux représentations des substances corporelles en Nouvelle-Guinée. L'Homme - Revue française d'anthropologie, 1990, 114, pp.101-120. halshs00007292

\section{HAL Id: halshs-00007292 \\ https://shs.hal.science/halshs-00007292}

Submitted on 7 Jun 2012

HAL is a multi-disciplinary open access archive for the deposit and dissemination of scientific research documents, whether they are published or not. The documents may come from teaching and research institutions in France or abroad, or from public or private research centers.
L'archive ouverte pluridisciplinaire HAL, est destinée au dépôt et à la diffusion de documents scientifiques de niveau recherche, publiés ou non, émanant des établissements d'enseignement et de recherche français ou étrangers, des laboratoires publics ou privés. 


\section{Considérations relatives aux représentations des substances} corporelles en Nouvelle-Guinée

In: L'Homme, 1990, tome 30 n¹14. pp. 101-120.

Citer ce document / Cite this document :

Bonnemère Pascale. Considérations relatives aux représentations des substances corporelles en Nouvelle-Guinée. In: L'Homme, 1990, tome 30 n¹14. pp. 101-120.

doi : $10.3406 /$ hom. 1990.369242

http://www.persee.fr/web/revues/home/prescript/article/hom_0439-4216_1990_num_30_114_369242 


\section{Abstract}

Reflections about the Representations of Bodily Substances in New Guinea. - Beliefs about bodily substances in some twenty New Guinea societies seem to form a system. After characterizing these substances and underlining their ambivalence, the author draws a parallel between representations linked to conception on the one hand and to ritualized homosexuality in male initiations on the other. It is shown that a woman must be symbolically present so that boys become men. This can be related to the fact that, in the ceremonial exchanges in big men societies, women play an essential mediating role in the transactions which need to be performed successfully in order to become an adult. Finally, the author tries to link modes of marriage and mortuary practices to representations of bodily substances through the principles of reciprocity and substitution.

\section{Résumé}

Considérations relatives aux représentations des substances corporelles en Nouvelle-Guinée. — Les croyances relatives aux substances corporelles dans une vingtaine de sociétés de Nouvelle-Guinée semblent former système. Après avoir caractérisé chacune de ces substances et souligné leur ambivalence, l'auteur établit un parallèle entre les représentations liées à la conception et celles qui le sont à l'homosexualité ritualisée lors des initiations masculines. Elle y montre la nécessaire présence symbolique d'une femme pour que les garçons deviennent des hommes. Ceci est alors rapproché du fait que dans les échanges cérémoniels des sociétés à big men, les femmes ont un rôle médiateur essentiel dans les transactions dont le succès assure le passage au statut d'adulte. Enfin l'auteur tente de relier types de mariage et pratiques mortuaires aux conceptions sur les substances corporelles au moyen des principes de réciprocité et de substitution.

\section{Zusammenfassung}

Uberlegungen zu den Vorstellungen uber Korpersubstanzen in Neuguinea. - Die Glaubensvorstellungen uber Korpersubstanzen in zwanzig Gesellschaften Neuguineas scheinen systematischen Charakter zu besitzen. Nachdem die Autorin jede dieser Substanzen charakterisiert und ihre Ambivalenz hervorgehoben hat, stellt sic eine Parallèle zwischen den Vorstellungen, die sich auf die Empfangnis, und solchen, die sich auf die in den mannlichen Initiationen ritualisierte Homosexualitat beziehen, her. Sie zeigt in diesem Zusammenhang die Notwendigkeit der symbolischen Prasenz einer Frau auf, damit aus Knaben Manner werden können. Dies wird dann mit der Tatsache in Annaherung gebracht, daB die Frauen in den Transaktionen des Zeremonialtausches der big me/7-Gesellschaften, dessen erfolgreiche Abwicklung den Übergang zum Erwachsenenstatus sichert, eine wesent-liche Vermittlerrolle spielen. Schliesslich versucht die Autorin mittels der Prinzipien Rezipro-zität und Substitution Heiratstypen und Totenbräuche mit den Vorstellungen uber Korper-substanzen in Verbindung zu bringen.

\section{Resumen}

Consideraciones relativas a las representaciones de las substancias cor-porales en Nueva Guinea. Las creencias relativas a las substancias corporales en una vein-tena de sociedades de Nueva Guinea parecen formar sistema. Tras haber caracterizado cada una de estas substancias y senalado su ambivalencia, el autor establece un paralelo entre las representaciones relacionadas a la conception y las que lo son a la homosexualidad ritualizada en el transcurso de las iniciaciones masculinas. Muestra la necesaria presencia simbólica de una mujer para que los jôvenes se hagan hombres. Esto es entonces relacionado con el hecho de que en los inter cambios ceremoniales de las sociedades con big men, las mujer es tengan un papel de mediador esencial en las transaciones cuyo exito asegura el paso al status de adulto. Para terminar, la autora trata de relacionar tipos de matrimonio y prácticas mor-tuorias a las concepciones sobre las substancias corporales por medio de los principios de reciprocidad y substitución.

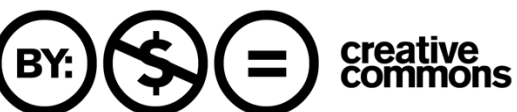




\title{
Considérations relatives aux représentations des substances corporelles en Nouvelle-Guinée
}

\begin{abstract}
Pascale BonNEMÈRE, Considérations relatives aux représentations des substances corporelles en Nouvelle-Guinée. - Les croyances relatives aux substances corporelles dans une vingtaine de sociétés de Nouvelle-Guinée semblent former système. Après avoir caractérisé chacune de ces substances et souligné leur ambivalence, l'auteur établit un parallèle entre les représentations liées à la conception et celles qui le sont à l'homosexualité ritualisée lors des initiations masculines. Elle y montre la nécessaire présence symbolique d'une femme pour que les garçons deviennent des hommes. Ceci est alors rapproché du fait que dans les échanges cérémoniels des sociétés à big men, les femmes ont un rôle médiateur essentiel dans les transactions dont le succès assure le passage au statut d'adulte. Enfin l'auteur tente de relier types de mariage et pratiques mortuaires aux conceptions sur les substances corporelles au moyen des principes de réciprocité et de substitution.
\end{abstract}

Depuis quelques années les ethnologues s'attachent aux représentations que les sociétés se font des substances produites et excrétées par le corps humain. Cela vaut notamment pour ceux qui étudient des sociétés de Nouvelle-Guinée (Biersack 1983 ; Herdt 1984a ; Hinton \& Mc Call 1983 ; J. F. Weiner 1982, entre autres). Cet intérêt tient sans doute au fait que, dans cette région du monde - mais sans doute aussi ailleurs - , des questions majeures de l'anthropologie ne peuvent être correctement posées si l'on néglige les conceptions que ces sociétés ont élaborées concernant le corps et ses productions (Jorgensen 1983:1). Notre objectif est ici de présenter et d'analyser des données susceptibles d'illustrer la proposition formulée par F. Héritier-Augé (1987 : 15) dans les termes suivants : "Les conceptions ethniques particulières touchant au corps et au mouvement des humeurs renvoient à des thèmes invariants dont l'organisation obéit à une structure logique commune. ")

C'est d'abord dans le domaine de la parenté que s'exprime l'importance des représentations des fluides corporels. En Nouvelle-Guinée, c'est en fonction de théories sur ces substances qu'est souvent légitimée l'appartenance d'un individu à un ou plusieurs groupes de parenté. Ainsi considère-t-on quelquefois le sperme comme un symbole de la continuité des générations parce qu'il est à 
l'origine des os d'un individu et que ceux-ci perdurent au delà de la mort. En tout état de cause, il est le fondement de l'identité patrilatérale (LiPuma 1979 : $43,1988: 48)$ et « le symbole des connections agnatiques en général » (Feil $1980: 29)$.

Il est également possible d'appréhender les théories sur le corps sous l'angle des relations entre les sexes et de leur conceptualisation. En Nouvelle-Guinée, les critères retenus pour affirmer une différence - qui se traduit le plus souvent par une inégalité - entre les hommes et les femmes sont d'abord d'ordre physiologique et prennent en compte les émissions corporelles. Il en résulte que cette opposition entre les individus des deux sexes est vécue comme irréductible : elle tient en quelque sorte à la nature des choses.

Enfin, lorsqu'on parle du corps, on parle de la vie. Tout au long de celle-ci, un individu subit dans son corps des transformations qui, pour les Néo-guinéens, résultent du mouvement, du transfert ou de la déperdition de diverses substances corporelles, parfois associées à des entités cosmologiques ou spirituelles - comme le soleil chez les Baruya (Godelier 1982:91) ou l'esprit baloma aux îles Trobriand (A. Weiner 1983 : 56 ; Young 1979: 99, 101). Ainsi, les conceptions indigènes concernant les humeurs forment le substrat des représentations de la production et de la constitution de la personne, et elles permettent d'aborder sous un angle complémentaire des questions telles que le mode d'affiliation des individus aux groupes de parenté, les rapports hommesfemmes, les représentations de l'individu et de l'identité sociale. C'est avant tout ces deux derniers champs qui seront traités ici.

Cet article est une étude comparative, à caractère provisoire en raison des informations encore lacunaires en ce domaine, des représentations ainsi élaborées par plusieurs sociétés de Nouvelle-Guinée (voir fig. 1). On tentera tout d'abord de définir les substances en cause et on se demandera si leur ensemble forme ou non système. Par ailleurs, les caractéristiques attribuées aux différents fluides corporels ne sont pas sans conséquences sur la vie sociale : elles déterminent en grande partie les pratiques rituelles, ainsi que les rapports entre les individus et les groupes. C'est pourquoi on présentera les divers contextes (« fabrication » d'un enfant, initiations masculines, mort) dans lesquels les humeurs jouent un rôle important. Enfin, on s'interrogera sur l'articulation des conceptions indigènes relatives aux substances corporelles et assimilées et des diverses pratiques accompagnant le mariage et les compensations mortuaires. Il s'agira de voir si des principes similaires ne sous-tendent pas les croyances et les activités dans ces différents domaines de la vie sociale.

Il pourra paraitre étonnant d'examiner quasi exclusivement les substances liées à la sexualité. En fait, il est difficile de savoir si cette restriction tient au silence des théories locales elles-mêmes sur des excrétions comme l'urine, la sueur, la morve, le pus ou les fèces, où à l'insuffisante des enquêtes. Quoi qu'il en soit, les informations concernant les émissions sexuelles sont, elles, nombreuses, peut-être parce qu'elles font partie des discours sur la caractérisation des sexes et la reproduction. Dans ce dernier champ, interviennent toutefois des 


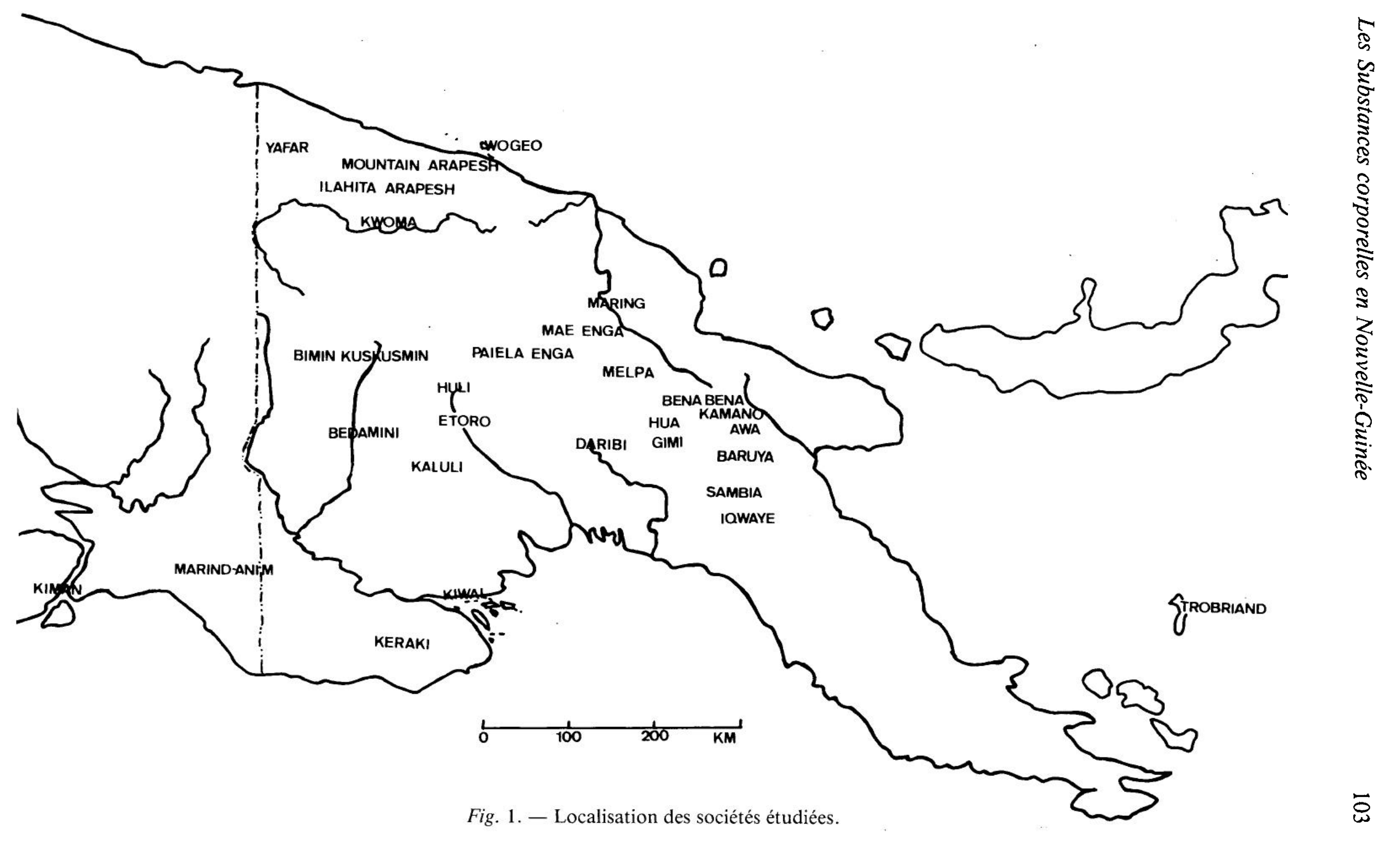


substances d'une tout autre nature (graisse de porc, jus de canne à sucre, lait de noix de coco, noix de pandanus, sang de porc et de casoar par exemple), que les représentations étudiées assimilent, par analogie, aux fluides liés à la reproduction. Parmi ceux-ci, le sperme, le sang menstruel et le lait maternel occupent des positions privilégiées.

\section{LA TRIADE SANG MENSTRUEL-SPERME-LAIT MATERNEL}

Une des croyances les mieux partagées dans les sociétés de notre échantillon concerne les caractéristiques, conçues comme opposées, du sang menstruel et du sperme. Dans leur très grande majorité - à l'exception des Etoro, des Maring et des Trobriandais (Kelly 1976 : 42 ; Buchbinder \& Rappaport 1976 : 21 ; Young 1979 : 98 ; A. Weiner 1983 : 138) -, ces sociétés affirment que le sang qui s'écoule régulièrement du corps des femmes est une source de pollution et de danger par excellence. A l'inverse, le sperme est à l'origine de la maturation des jeunes hommes et de la force masculine. Ces croyances qu'un examen plus poussé nuancera - déterminent un certain nombre de pratiques sociales et marquent l'opposition entre les sexes.

C'est par exemple en raison du risque de pollution par le sang menstruel que les hommes et les femmes ne cohabitent pas et a fortiori n'ont pas de rapports sexuels pendant la menstruation ${ }^{1}$, ou que les femmes ne cuisinent pas pour leur mari (Godelier 1982 : 99 ; M. Strathern $1972: 166$ ), ou encore que le sang féminin est vecteur de sorcellerie (Meigs 1976 : 396 ; M. Strathern 1972 : 173). Dans les cas les plus extrêmes (Paiela-Enga), la femme qui a ses règles doit éviter tout contact avec son propre sang menstruel : pour se protéger, elle accomplit certains rites et prend d'immenses précautions pour se débarrasser de ses tampons de mousse végétale (Biersack 1980 : 180-182). Les femmes biminkuskusmin sont susceptibles d'être contaminées par du sang menstruel au même titre que les hommes (Poole 1982 : 113) et chez les Yafar, parmi les personnes qui en craignent la contamination, figurent les propres parents de la femme menstruée (Juillerat 1986 : 253-254). Néanmoins, dans la majorité des cas, ce sont surtout les hommes qui sont les victimes potentielles d'un contact avec cette substance - comme chez les Baruya, Huli, Kiwai, Mae-Enga, Melpa (Godelier 1982 : 99, 106 ; Glasse 1974 : 82 ; Landtman 1927 : 237-238 ; Meggitt 1964 : 207 ; Strathern \& Strathern 1969 : 152). Il arrive qu'une catégorie d'hommes soit particulièrement exposée, par exemple les adolescents et les guerriers chez les Gimi (Gillison 1983 : 34). Dans certaines sociétés, faire l'amour avec une femme menstruée - Awa (Newman \& Boyd 1982 : 267) — ou consommer de la nourriture qui a été en contact avec du sang menstruel - Hua (Meigs 1976 : 395-396) - est l'une des causes principales de la maladie dénommée « grossesse masculine », caractérisée par un gonflement prononcé du ventre.

C'est en vertu de son pouvoir de faire croître et de rendre fort que le sperme 
doit être dispensé aux jeunes initiés - chez les Baruya, Bedamini, Etoro, Kaluli, Kiwai, Marind-Anim et Sambia (Godelier 1982 : 91 ; Sorum 1984 : 324 ; Kelly 1976 : 45 ; Schieffelin 1976 : 126 ; Landtman 1927 : 237 ; Van Baal $1966: 143$; Herdt $1982: 82$ ) - ou que la femme enceinte doit continuer à en recevoir pour permettre au fœetus de se développer - Baruya, Gimi, MaeEnga, Melpa, Sambia ou Yafar (Godelier 1982 : 90 ; Gillison 1986 : 50 ; Meggitt 1965 : 164 ; M. Strathern 1972 : 168 ; Herdt 1981 : 196 ; Juillerat 1986 : 246).

A cette opposition fondamentale entre le sang menstruel et le sperme s'en ajoutent d'autres, moins visibles. Ainsi, le sang menstruel est considéré un peu partout en Nouvelle-Guinée comme une humeur que l'on est tenté de qualifier d' " innée »: c'est de manière naturelle qu'il est présent dans le corps des femmes et il n'a pas besoin de stimulant ou d'apport extérieur pour se manifester (J. F. Weiner 1987 : 263). Sur cette croyance est calquée celle de la maturation spontanée des jeunes filles, dont les Awa, Bimin-Kuskusmin, Kaluli, Sambia, parmi d'autres, pensent qu'elles deviennent des femmes sans intervention d'aucune sorte (Newman \& Boyd 1982 : 282 ; Poole 1982 : 115 ; Schieffelin $1982: 162$; Herdt $1982: 55)^{2}$. A l'inverse, le sperme ne semble pas posséder une grande capacité d'auto-production. De là résulte la nécessité d'en fournir aux jeunes garçons (Herdt 1981 : 218). Comme en corollaire, le sperme n'existe qu'en quantité limitée et les hommes doivent veiller à ne pas en dépenser de manière abusive, sous peine de dépérir rapidement (Herdt 1984a : 176). En tout état de cause, le liquide séminal est une substance transmissible, ce que n'est jamais le sang menstruel. En outre, à défaut de pouvoir s'auto-produire, il est doté d'une capacité de transformation que n'a pas le sang menstruel. On verra par exemple que dans le corps des femmes le sperme devient quelquefois du lait maternel. En revanche, le sang menstruel ne peut jamais se transformer en une autre substance ${ }^{3}$.

Le lait maternel est le troisième terme de cet ensemble. C'est une humeur dépourvue d'autonomie et souvent placée dans une relation d'équivalence avec le sperme (Herdt 1984a : 176). D'abord, elle en est quelquefois la simple transformation, le sperme devenant du lait dans le corps féminin, comme chez les Bimin-Kuskusmin et les trois groupes anga de notre échantillon, Baruya, Iqwaye et Sambia (Poole 1982 : 113 ; Godelier 1982 : 98 ; Mimica 1981 : 102 ; Herdt 1984a : 181-182). Ensuite, la consommation des mêmes nourritures est réputée produire soit du sperme soit du lait, selon le sexe de ceux qui les absorbent (Herdt 1984a : 181). Enfin, le même rôle nourricier est accordé aux deux substances. En outre, dans les nombreuses sociétés néo-guinéennes où le sperme et le lait sont ainsi fortement associés, le sang menstruel constitue alors un pôle singulier, opposé à cette paire.

Rarement mentionnés, les fluides vaginaux ont néanmoins parfois une place dans l'ensemble de ces représentations. C'est du côté du sperme qu'on les trouve rangés : ils sont « de la même matière que le sperme », affirment les Gimi (Gillison 1986 : 49). 


\section{DES RELATIONS QUI FORMENT SYSTÈME ?}

Deux relations - auxquelles on peut éventuellement en ajouter une, secondaire, caractérisée par une forte contiguité symbolique - suffisent dans une large mesure pour organiser cet ensemble de substances en un système : il s'agit des relations d'opposition/complémentarité et d'homologie.

Des substances placées dans un rapport d'opposition présentent des caractères contrastés. L'exemple le plus net est sans doute celui du sperme et du sang menstruel. Au premier abord, tout les différencie : couleur, origine (corps masculin ou féminin), quantité (limitée ou illimitée), effets (nourriciers ou destructeurs - ou du moins ambigus). En Nouvelle-Guinée, ces deux humeurs sont donc difficilement conciliables. Pourtant, elles sont complémentaires car c'est de leur combinaison que se forme un nouvel être humain. Des principes en opposition sont donc nécessaires à la perpétuation de la vie. Il arrive également que le sperme et le lait maternel soient conçus comme opposés. Les Mae-Enga, par exemple, pensent que des rapports sexuels qui auraient lieu pendant l'allaitement conduiraient à la contamination du lait maternel par le sperme (Meggitt 1965 : 164). Ici, il n'existe pas même de complémentarité ; les deux substances ne supportent pas la mise en contact et on a là l'exemple extrême, bien que rarement attesté, de la pure relation d'opposition.

La relation d'homologie entre deux éléments suppose qu'on leur attribue des fonctions, voire une origine, semblables ou proches. En Nouvelle-Guinée, c'est le plus souvent le cas du sperme et du lait maternel. Ces substances ont une origine commune mais se présentent sous deux formes distinctes en raison de la différence anatomique entre l'homme et la femme. Ainsi, les Daribi conçoivent le corps humain comme un ensemble de deux réseaux de " tubes » dont l'un produit le sperme chez l'homme, le lait maternel chez la femme, l'autre étant le système des veines et artères qui transportent le sang (Wagner 1983 : 76). Chez les Iqwaye, la moelle osseuse se transforme chez les femmes en lait et non en sperme en raison de l'absence de pénis (Mimica 1981 : 102). En outre, des aliments semblables - noix de pandanus chez les Sambia, graisse de porc chez les Daribi (Herdt 1984a : 182 ; Wagner $1977: 628$ ) - sont en général ingérés pour produire soit du sperme, soit du lait, selon le sexe du consommateur. Tous ces phénomènes font penser que le sperme et le lait maternel sont souvent conceptualisés en Nouvelle-Guinée comme des substances homologues. Néanmoins, il apparaît, à l'analyse des commentaires d'hommes baruya et sambia, que leur homologie est présentée ici d'une façon quelque peu différente. Selon les premiers en effet, "le lait de leurs épouses n'est que du sperme, transformé en substance nourricière pour les enfants » (Godelier 1982 : 98), et selon les seconds, "le corps féminin est comme un transformateur biologique de sperme » (Herdt 1984a : 181), ce qui impliquerait que le lait maternel ne soit qu'une forme transformée de sperme. On serait alors tenté de dire que ces deux fluides corporels ne sont considérés que comme les deux formes d'une même 
substance. Il semblerait donc que la relation particulière qu'entretiennent dans ces deux groupes anga le sperme et le lait maternel soit difficilement formalisable selon une relation d'homologie.

Cela dit, si les Baruya semblent considérer le lait maternel comme simplement et toujours produit à partir de sperme, les Sambia ont, quant à eux, une conception qui diffère légèrement. D'abord, la consommation par une femme de noix de pandanus produit du lait maternel sans intervention aucune de liquide séminal (Herdt 1981 : 110) ; ensuite, de nombreuses femmes sambia affirment que leur corps produit du lait maternel " naturellement » (Herdt 1984a : 182). Si la notion d'homologie n'est pas tout à fait adéquate pour caractériser la relation entre sperme et lait maternel dans les deux groupes anga en question - particulièrement chez les Baruya -, on peut la retenir dans les autres cas évoqués.

Si l'on veut déterminer la relation qui unit humeur corporelle et nourriture, l'entreprise est plus délicate. Tout en se rapprochant de l'homologie, elle s'en distingue par le fait qu'elle associe des éléments de nature différente et qu'elle est non réciproque. En effet, c'est toujours la nourriture qui se transforme dans le corps humain en une substance ou qui en déclenche la production, l'inverse n'étant que rarement attesté. On remarquera néanmoins que les femmes yafar s'enduisent de sperme pour assurer une meilleure production de sagou (Juillerat 1986 : 257), sans qu'on puisse affirmer pour autant que le sperme se métamorphose en sagou. Pour préciser la relation que certaines sociétés de Nouvelle-Guinée établissent entre des éléments de nature différente, on pourrait peut-être parler de remplacement, ou, mieux, de contiguïté symbolique. Dans une société comme les Sambia, des noix de pandanus sont consommées pour véritablement remplacer le sperme ou le lait maternel (Herdt 1984a : 182). L'analyse des tabous alimentaires permet de dégager indirectement d'autres relations de contiguïté : par exemple chez les Hua, entre le sang menstruel et certains aliments de couleur rouge, prohibés pendant la durée des menstrues, par crainte de provoquer une hémorragie incontrôlable (Meigs 1984 : 82-83). De même, chez les Yafar, la femme menstruée ou prête à accoucher s'interdit de consommer du pandanus rouge pour éviter un excès de sang (Juillerat $1986: 247$ n. 8, 250). Dans ces deux exemples, l'aliment rouge augmenterait dangereusement la quantité de sang dans le corps de la femme.

Pour résumer, on dira que dans les sociétés considérées deux relations principales, l'opposition et l'homologie - auxquelles on peut adjoindre celle de remplacement (ou contiguïté) - semblent mettre en système les substances corporelles. Le remplacement serait une sous-catégorie du rapport d'homologie plutôt qu'une relation véritablement autonome. La forme graphique suivante rend assez bien compte des relations que les substances sexuelles et assimilées entretiennent entre elles dans la société sambia, pour laquelle les données sont suffisantes. 


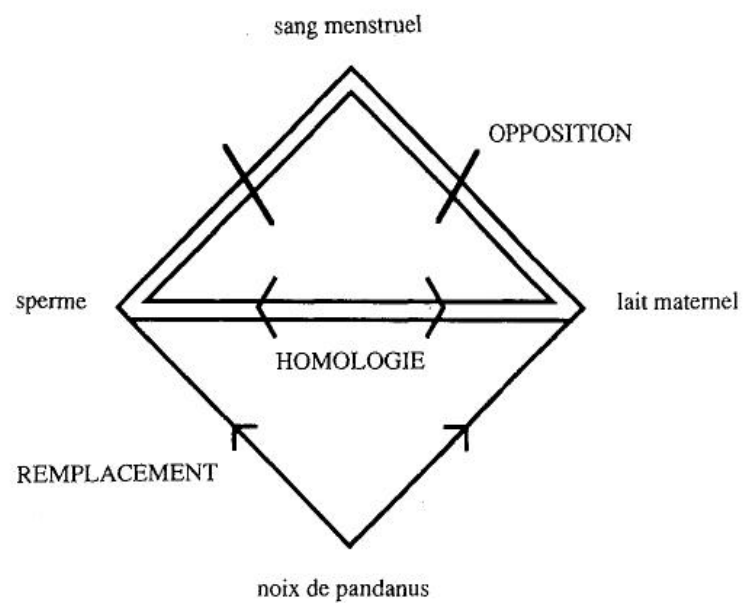

Fig. 2. Relations entre les substances ches les Sambia.

Il reste à démontrer que si l'une des relations au sein de cet ensemble changeait, toute la composition en serait affectée, et l'on pourrait alors parler de système.

\section{UN ENSEMBLE FINALEMENT MARQUÉ D'AMBIVALENCE}

A y regarder de plus près, et si l'on s'attache maintenant au discours sur les propriétés des substances plutôt qu'aux relations qu'elles entretiennent entre elles, on est frappé par le fait que ces propriétés sont marquées d'ambivalence. Il n'est pas possible de qualifier certaines substances de positives et d'autres de négatives. Elles ne sont en effet jamais seulement l'un ou l'autre, mais comportent les deux qualités, que les sociétés de Nouvelle-Guinée manipulent au gré des circonstances.

Ainsi en est-il du sang menstruel, élément polluant par excellence qui, dans certaines sociétés et sous certaines conditions, se trouve pourvu de qualités curatives - il sert d'antidote chez les Mountain Arapesh (Mead 1940 : 345) ou même érotiques chez les Gimi (Gillison 1980 : 153). Chez les Yafar, les hommes récupèrent un peu du sang de leurs femmes et s'en servent dans leurs magies de chasse (Juillerat 1986 : 256). Dans un sous-groupe enga - les Laiagam - , les femmes placent un peu de leur sang menstruel, préalablement enveloppé, dans le creux d'un arbre pour assurer la prospérité des cochons et des récoltes (Gray 1973 : 116, cité in Feil 1987 : 224). Plus largement, la menstruation est le signe de la fertilité des femmes et c'est dans ce domaine et celui de la vie en général que les sociétés de Nouvelle-Guinée situent l'aspect positif du pouvoir du sang menstruel.

Le sperme connaît un traitement du même ordre, mais inverse : d'élément largement positif, il devient quelquefois vecteur de danger. Chez les Mountain Arapesh, les Kamano et les Melpa, il est utilisable en sorcellerie (Mead 1940 : 355 ; Berndt 1962 : 214 ; M. Strathern 1972 : 166). Selon les Huli, les Kamano 
et les Mae-Enga (Glasse $1968: 62$ n. 1 ; Mandeville $1979: 234$; Meggitt 1965 : 164), il est susceptible de contaminer le lait maternel. Chez les Iqwaye, le sperme qu'une femme adultère reçoit d'un amant est porteur de maladie grave pour le mari (Mimica 1981 : 143). Chez les Yafar, le simple emploi du mot sperme par une femme s'adressant à un homme est une insulte grave (Juillerat 1986 : 240). A ce propos, on remarquera que l'aspect linguistique, si l'on peut dire, des substances corporelles n'a pas été évoqué ici. Les mots qui les désignent sont très souvent l'objet d'interdits car ils sont presque autant chargés de dangers que les substances elles-mêmes. De même, dans certaines circonstances, le lait maternel perd ses qualités nourricières pour devenir poison ou source de pollution - chez les Baruya, Huli et Ilahita Arapesh (Godelier 1982 : 97 ; Goldman 1983 : 94 ; Tuzin 1982 : 337). A l'échelle de la Nouvelle-Guinée, le système de représentations de ces substances n'est donc en aucune façon constitué d'éléments figés. Il est dynamique, et les transformations ou les différences de valeur accordées à ses éléments en modifient les relations.

\section{LES SUBSTANCES DANS LE CYCLE DE VIE}

Les croyances concernant les humeurs corporelles déterminent nombre de pratiques rituelles et de comportements quotidiens. De la conception à la mort en passant par les initiations, la totalité du cycle de vie fait l'objet de représentations et de pratiques sociales dans lesquelles interviennent des idées sur les substances émises par le corps. Ici, les opérations en jeu peuvent se résumer en trois mots clefs : transfert simple, transformation, substitution sans transformation. Ainsi a-t-on déjà rencontré, chez les Baruya, Etoro ou Sambia, le transfert de sperme entre des jeunes hommes encore célibataires et des garçons en première phase d'initiation (Godelier 1982: 91 ; Kelly $1976: 41$; Herdt 1981 : 204) ; on a également vu la transformation que subissent certaines nourritures ou fluides sexuels à l'intérieur du corps. Pour illustrer cette opération, reprenons simplement l'exemple des noix de pandanus, qui produisent du sperme dans le corps masculin, du lait maternel dans le corps féminin, et celui du sperme qui, dans le corps des femmes baruya, devient du lait maternel. C'est ainsi que cette transformation met en jeu des substances placées dans les rapports précédemment décrits d'homologie et de remplacement.

Dans ces exemples, le corps n'est pas un lieu vide où les capacités de transformation des éléments absorbés s'actualiseraient de manière autonome. Il possède un " appareillage » anatomique spécifiquement destiné à cette opération, comme chez les Baruya et les Sambia (Godelier 1982 : 98 ; Herdt 1984a : 181182). La conception d'un enfant est, elle aussi, l'occasion d'une transformation. Que celle-ci s'opère par le mélange du sperme et du sang menstruel — chez les Hua et Sambia par exemple (Meigs 1976 : 395 ; Herdt 1981 : 195) —, ou par la combinaison du sperme et des liquides vaginaux de la femme - Gimi et Wogeo (Gillison $1986: 49$; Hogbin 1943 : 287) —, ou encore par l'action 
unique, ou principale ${ }^{4}$ du liquide séminal - Baruya, Bimin-Kuskusmin, Maring, Melpa (Godelier 1982 : 90 ; Poole 1984 : 196 ; LiPuma 1981 : 275 ; M. Strathern 1972 : 42) -, le fœtus résulte d'un changement d'état. Le mélange de ces différentes humeurs dans la matrice maternelle aboutit à la création, par transformation, d'une entité de nature différente, l'embryon.

La substitution sans transformation est plus difficile à concevoir. On peut parler de substitution lorsqu'une équivalence est clairement établie entre de la nourriture, ou même des objets, et la personne, constituée de substances. Ainsi, à la mort d'un de leurs membres, de nombreuses sociétés de NouvelleGuinée distribuent du porc ou du gibier - ou encore des richesses (coquillages, sel végétal, etc.) - aux parents maternels du défunt. Selon l'interprétation la plus courante, il s'agirait d'une compensation versée à ceux des parents que l'on considère comme responsables de la chair et du sang d'un individu, en l'occurrence les maternels, dans la majorité des cas - par exemple, Etoro, Huli, Iqwaye, Melpa et Paiela-Enga (Kelly 1977 : 91 ; Goldman 1983 : 73 ; Mimica 1981 : 43, 94, 101 ; Strathern \& Strathern 1969 : 141 n. 3 ; Biersack 1983 : 93, 96). On substitue alors indéniablement de la nourriture ou des richesses au corps humain.

Cette brève illustration montre que la « régulation nécessaire des fluides corporels »- dont F. Héritier-Augé (1987 : 12) avance l'universalité probable $-\mathrm{s}$ 'applique tout à fait ici : les sociétés de Nouvelle-Guinée ont organisé le champ des substances sexuelles et assimilées en des systèmes de représentations qui régissent les comportements dans divers domaines de la vie sociale.

\section{D'UN PARALLÈLE ENTRE LA CONCEPTION ET LES INITIATIONS}

Lorsqu'on examine les différents rituels initiatiques en Nouvelle-Guinée, on s'aperçoit qu'il existe une incompatibilité totale entre la fellation et les saignées péniennes. Ce dernier procédé - qui dans notre échantillon concerne les Arapesh, Bena-Bena, Kamano et Kwoma - consiste à faire s'écouler du sang du pénis en insérant des tiges végétales dans l'urètre ou en l'incisant. Il se pratique collectivement à l'adolescence, puis se maintient à l'âge adulte en tant qu'acte individuel. Ses objectifs sont multiples : préparation à une activité rituelle, stimulation de la croissance des garçons, expulsion du mauvais sang provenant de la mère, maintien de la force physique (Mead 1940 : 347 ; Williamson 1983 : 18). Comment alors interpréter l'incompatibilité absolue de la fellation et des saignées péniennes qui visent pourtant des buts complémentaires ?

Le sang qui s'écoule lors des saignées péniennes étant considéré comme du sang menstruel (Herdt 1981:245; Hogbin 1970: 88), on peut rapprocher cette incompatibilité de l'interdiction de poursuivre des rapports homosexuels après le mariage : de même qu'est "impensable » le contact d'un jeune garçon avec le pénis d'un homme contaminé par le sexe d'une femme, de même le contact avec un pénis dont a coulé du sang «menstruel » constituerait une menace pour la santé de l'initié. 
Sans entrer dans le détail des nombreuses « manipulations » - réelles ou imaginaires - opérées sur les substances corporelles dans cette région du monde, on ne peut manquer d'être frappé par le parallélisme entre les pratiques homosexuelles ritualisées - fellation, sodomie et onction - et les règles matrimoniales, autrement dit entre initiation et conception. Dans l'homosexualité, les partenaires ne sont pas choisis au hasard : ce sont généralement des affins. Chez les Etoro et les Sambia, le mari d'une sœur aînée ${ }^{5}$ est un partenaire particulièrement indiqué pour un jeune garçon (Kelly 1976 : 52 n. 6 ; Herdt $1981: 238,1984 a: 193)$. Les Baruya interdisent à tout parent en ligne paternelle ou maternelle d'un initié d'être son inséminateur (Godelier 1982 : 94). Il en est de même chez les Kaluli (Schieffelin 1976 : 124). Chez les Bedamini et les Keraki, le partenaire d'un garçon est choisi parmi des hommes appartenant à la moitié opposée de celui-ci, c'est-à-dire parmi l'un des clans où il trouvera épouse (Sorum 1984 : 323 ; Williams 1936 : 194). En bref, il semble que les règles de prohibition qui pèsent sur l'homosexualité rituelle suivent celles qui gouvernent les mariages. L'idée sous-jacente paraît concerner la façon dont un être humain est « fabriqué ». On peut en effet mettre en parallèle la grossesse, lors de laquelle, dans la plupart des systèmes de croyance néoguinéens, le sperme intervient dans le développement du fœtus, et les initiations où le sperme permet la croissance et la maturation des jeunes garçons. En outre, si l'on en croit le discours des intéressés eux-mêmes, il n'est pas déraisonnable de concevoir les initiations comme une re-naissance, effectuée au sein d'un monde exclusivement masculin (voir par exemple Godelier 1982 : 91 ; Herdt 1987 : 116 ; Serpenti 1984 : 303).

La grossesse supposant l'existence d'un lieu intermédiaire - la matrice maternelle -, on pourrait postuler que les systèmes de représentations de la maturation des garçons supposent également la nécessité d'un intermédiaire féminin. Or, la relation entre un initié et son beau-frère (Etoro, Sambia) ou n'importe quel affin (Kaluli) implique un médiateur, en l'occurrence une femme. De même, dans les sociétés où c'est un oncle maternel, réel ou classificatoire, qui donne son sperme à un initié - chez les Marind-Anim ou les Kimam-Papuans (Van Baal 1984 : 132 ; Serpenti 1965 : 163, 1984 : 304), - le lien entre ces deux individus passe également par une femme qui est la mère du garçon et la sœur de l'adulte. Ne peut-on alors considérer que dans ces sociétés l'initiation s'apparente non seulement à une naissance mais aussi à une grossesse. Les hommes reproduiraient à la puberté ce qui s'est passé dans le ventre de la mère (Moisseeff 1987 : 132). En apparence, cette re-naissance s'opère en dehors de toute influence féminine ; en fait, la nécessité d'un intermédiaire féminin se trouve réaffirmée à travers le choix de l'homme responsable de la maturation d'un initié. Pour permettre à la re-naissance masculine d'avoir lieu, puis à la croissance de se réaliser, la transmission de sperme entre les deux hommes que sont l'initié et son initiateur doit en quelque sorte être fondée sur leur lien à une même femme. A cet égard, il est tout à fait remarquable que chez les Iqwaye, où les partenaires homosexuels sont dans une relation de frères 
classificatoires (aîné et cadet), le mythe de la création du premier homme « nie la nécessité de la femme dans la reproduction sexuelle »: le premier Iqwaye se crée tout seul, au moyen d'une auto-fellation (Mimica 1981 : 202, 205). Cela confirmerait a contrario l'existence d'une relation profonde entre le monde de la conception et celui des initiations ${ }^{6}$.

Pour affirmer ce parallélisme, on peut mettre l'accent sur les similitudes des représentations concernant le sperme dans les domaines de la conception et des initiations. En effet, à l'exception, semble-t-il, des Iqwaye (Mimica 1981:100), l'ensemble des sociétés qui insistent sur le besoin de sperme pour que les garçons parviennent à l'âge adulte conçoivent également cette substance comme essentielle à la croissance du fœtus et « prescrivent » logiquement la continuation des rapports sexuels pendant une partie au moins de la grossesse (Godelier 1982 : 90 ; Herdt 1981 : 196 ; Schieffelin 1976 : 124 ; Sorum 1984 : 322 : Williams $1936: 172)^{7}$.

Un autre trait vient peut-être à l'appui de l'hypothèse de la nécessité d'un intermédiaire féminin pour rendre effective la croissance masculine : c'est la croyance répandue selon laquelle, dans les temps mythiques, les femmes détenaient les objets utilisés dans les initiations masculines - thème récurrent chez les Anga par exemple. Ainsi les Baruya révèlent-ils aux initiés que les flûtes qui jouent un rôle essentiel dans les rituels secrets masculins furent inventées par les femmes (Godelier 1982 : 117-118). Dans le même ordre d'idées, chez les Sambia, le partenaire adulte se déguise en femme lors de la cérémonie où les flûtes sont présentées aux initiés en même temps qu'on leur impose de consommer du sperme (Herdt 1982 : 78-79). Force est de constater que ces diverses pratiques s'analysent mieux si l'on postule la nécessité - farouchement cachée aux femmes - d'un médiateur féminin pour faire renaître les garçons au monde des hommes.

Si l'on voulait aller encore plus loin et tenter un parallèle entre les sociétés à homosexualité ritualisée et celles qui mettent l'accent sur les échanges cérémoniels de richesses - notamment les sociétés à big men -, on pourrait reprendre les arguments de D. Feil $(1978,1984)$ concernant la position intermédiaire des femmes dans les transactions entre partenaires d'échange. Mais avant de rapprocher les deux situations, une remarque s'impose : elle concerne l'incompatibilité de fait attestée dans les sociétés de Nouvelle-Guinée entre les initiations masculines ${ }^{8}$ et les grands échanges cérémoniels. Compte tenu de cette situation de fait, on a postulé que ces derniers occupaient, là où ils existaient, une position fonctionnellement équivalente à celle qu'ont ailleurs les initiations. Dans tous les cas, ces deux institutions sont des « faits sociaux totaux »; elles expriment à elles seules les caractéristiques essentielles des sociétés où elles apparaissent.

De plus, les objets - porcs et coquillages - qui circulent lors des grands échanges cérémoniels sont bien plus que de simples objets. Ces échanges se présentent très souvent comme l'extension de dons compensatoires versés lors d'un mariage ou d'un décès pour remplacer une vie. Il s'agit là d'une véritable 
substitution : à la place d'un homme ou d'une femme, on donne de la nourriture ou des richesses.

Ainsi, que l'on manipule des substances directement dérivées du corps humain, comme lors des initiations, ou que l'on échange des coquillages, il semble bien que ce qui circule dans les deux cas soit des principes de vie. Si grands échanges cérémoniels et initiations masculines se révèlent incompatibles, c'est probablement qu'ils ont une signification symbolique similaire. Que l'on décèle alors, dans les deux phénomènes, des traits structuraux communs n'aurait rien pour surprendre.

Si l'on reprend l'argument de D. Feil $(1980: 23,24)$ selon lequel les femmes, en reliant des hommes par l'institution du mariage, rendent les échanges possibles, qu'elles seules créent les « voies » du tee - le grand échange cérémoniel des Enga - et déterminent le contenu des relations d'échange (Feil 1978 : 221), on voit que les femmes ont dans les grands échanges une position intermédiaire que l'on retrouve, semble-t-il, à un niveau symbolique dans les initiations masculines.

D'où une question supplémentaire : les échanges cérémoniels ont-ils, à l'instar des initiations, un rôle dans la formation de l'identité masculine ? Si l'on en croit Modjeska (1982 : 64), la réponse serait plutôt positive puisqu'il affirme que dans les sociétés à initiations « les hommes sont ' faits' par la société masculine », alors que dans celles à big men " ils se font eux-mêmes à travers la production et l'échange ». Sans adhérer totalement à cette formule, je rappellerai que dans les sociétés à big men, les jeunes gens sont encouragés par leurs pères à acquérir leur autonomie dans les échanges avant le mariage (Feil 1984 : 138 ; Lederman 1986 : 74 ; A. Strathern 1971 : 196). L'acquisition de partenaires d'échange ést donc ici une condition d'accès au statut d'homme.

Ce que ces faits prouvent probablement, c'est que devenir homme sans l'intervention des femmes est chose impossible dans toutes ces sociétés. On peut postuler que cela tient aux capacités procréatrices des femmes. En effet, quelle que soit la part accordée aux hommes dans la conception et la croissance du fœtus, c'est dans le ventre des femmes que la vie voit le jour. Ce fait indéniable se trouve réaffirmé dans les pratiques initiatiques ou leurs équivalents fonctionnels alors même que tout, en apparence, est fait pour le nier ou l'occulter.

\section{RÉCIPROCITÉ OU SUBSTITUTION}

En conclusion, essayons de dégager certains des principes à l'œuvre dans ces systèmes de représentations et de les rattacher à d'autres traits de l'organisation sociale. En d'autres termes, existe-t-il des associations préférentielles entre certaines croyances relatives aux humeurs et certaines pratiques culturelles : types de mariages ou coutumes funéraires, par exemple ?

Plusieurs auteurs vont dans ce sens. On a notamment fait remarquer (Gode- 
lier $1982: 271$; Herdt 1984b : 70 ; Lindenbaum $1984: 345$; J. F. Weiner 1982 : 29 n. 9) que les sociétés qui pratiquent l'homosexualité lors des initiations tendent à régler les mariages selon le principe de l'échange direct de femmes, en l'occurrence l'échange des sœurs. Parallèlement, on a noté que ces sociétés sont de petite taille, que la guerre y jouait jadis un rôle fondamental et qu'elles ne connaissent pas d'échanges cérémoniels de grande ampleur.

Pour G. Herdt (1984a : 193), R. Kelly (1976:52 n. 6) et S. Lindenbaum (1984: 344), les règles de mariage et d'homosexualité se combinent et se soutiennent mutuellement. Les relations entre affins seraient ainsi réaffirmées par les échanges de sperme qui ont lieu entre beaux-frères. La logique interne de ces deux pratiques passerait par le principe de réciprocité (Lindenbaum 1987 : 227). Pour être plus exact, on pourrait même parler de réciprocité à l'identique.

D'autres sociétés (dans notre échantillon : les Mae-Enga, Maring ou Melpa) font usage du principe de substitution, qui apparaît alors à l'œuvre dans tous les domaines de la vie sociale. Du mariage avec compensation matrimoniale au don de richesses à la mort d'un individu, en passant par l'équation entre nourriture et substances sexuelles, partout dans ces sociétés on substitue des richesses ou des aliments (notamment du porc) à la personne ou aux produits du corps humain.

S'il est vrai que le principe de substitution est plus ou moins accentué et étendu à divers aspects de la vie sociale selon les groupes considérés, il serait faux de croire que ceux qui pratiquent l'homosexualité - où du sperme produit du sperme (pas de substitution) - et l'échange des sœurs - où une femme vaut une femme - ne font aucun usage de ce principe. Il suffit de citer le cas des Sambia, qui, tout en constituant le type même de la société fondée sur le principe de la "réciprocité à l'identique », admettent l'équation entre nourriture et substances corporelles lorsqu'ils consomment des noix de pandanus pour augmenter leur stock de sperme (Herdt 1984a : 182). On peut aussi mentionner les Etoro, les Iqwaye et les Kaluli, chez qui on observe une homosexualité ritualisée et qui connaissent pourtant l'usage de compensations matrimoniales.

En bref, même s'il existe une tendance incontestable, dans les sociétés caractérisées par le principe de réciprocité, à faire un usage très limité de celui de substitution, il apparaît clairement que ces deux principes ne s'excluent pas totalement. On a vu notamment que le principe de substitution n'est pas la prérogative de quelques sociétés seulement. Il reste alors à tenter de répondre à la question posée par S. Lindenbaum (1984) : pourquoi, pour se marier ou après un décès, certaines sociétés " manipulent 》 des objets qui relèvent du corps, alors que d'autres font usage de richesses (produits détachés du corps) ? Pour cela, il faudrait articuler le champ des représentations concernant les substances et celui des pratiques matrimoniales et des compensations mortuaires. Au préalable, on doit se demander si le concept de substitution est applicable de la même façon dans ces divers domaines et s'il recouvre des réalités du même ordre. Sans doute le principe de substitution doit-il exister dans les 


\author{
croyances relatives aux substances corporelles et assimilées - qui concernent \\ donc la personne - pour qu'il intervienne aussi dans le champ du mariage ou \\ de la mort.
}

Décembre 1988

NOTES

1. Il n'est peut-être pas inutile de noter que ce tabou sur les relations sexuelles pendant les règles existe aussi dans les trois sociétés de l'échantillon (Etoro, Maring, Trobriandais) qui n'insistent pas particulièrement sur le caractère polluant du sang menstruel. En revanche, chez elles, les actes de sorcellerie ne font jamais appel à cette substance (KELly $1976: 39-40$; HeAley 1985 : 164 n. 10 ; A. WEINER $1983: 138$ ).

2. A l'exposé des conceptions baruya, on pourrait ici formuler une objection : «Si c'est une fille, Lune doit de nouveau la percer pour l'ouvrir aux hommes et la rendre fécondable. Si c'est un garçon, il faut que les hommes et le Soleil le disjoignent des femmes, le reprennent aux femmes et le fassent naître une seconde fois parmi eux » (GODELIER 1982 : 93). Ici, la symétrie entre maturation féminine et masculine semble parfaite. Néanmoins il est clair que, dans l'esprit des gens, une intervention dans le développement physique des hommes est absolument indispensable, alors que la menstruation est un phénomène sans doute induit par la lune mais sur lequel les êtres humains n'ont pas de prise. D'ailleurs, c'est seulement après ses premières règles qu'une jeune fille est soumise aux rituels d'initiation, alors que pour les hommes ces rites sont une (pré-) condition nécessaire à leur maturation physique.

3. La modification de nature que subissent certaines substances - en général sperme et sang menstruel mélangés ensemble - pour devenir un embryon est d'un ordre différent. Elle est soumise à des règles autres, nées précisément du fait qu'il s'agit d'un mélange qui engendre une entité toute différente, et non de la simple transformation d'une unique substance en une autre.

4. Le rôle primordial du sperme est souvent lié à la nécessité d'accumuler celui-ci dans la matrice féminine pour que la conception soit effective. Dans l'échantillon considéré, les Iqwaye sont les seuls à penser qu'un unique coït suffit à implanter le fœtus (Mimica $1981: 110$ ).

5. Il s'agit en fait du futur mari d'une sœur aînée, puisqu'en règle générale - à l'exception semble-t-il des Marind-Anim (VAN BAAL 1984 : 136) - des hommes mariés ne peuvent servir de partenaires aux jeunes garçons.

6. Herdt semble dire la même chose, tout en considérant le problème sous un angle différent - celui des échanges entre groupes - lorsqu'il écrit que « le flux de sperme reflète les transactions matrimoniales entre groupes $\leadsto(1984 a: 193)$. On notera que c'est ici une réflexion sur les représentations des substances - mettant en parallèle la conception (plutôt que le mariage) et les initiations - qui conduit à la même constatation.

7. Notons cependant que toutes les sociétés qui accordent un rôle important au sperme dans la « fabrication » d'un enfant (conception et grossesse) ne pratiquent pas pour autant l'homosexualité ritualisée (Gimi par exemple). Certaines ne connaissent pas même de « véritables » initiations masculines, tels les Melpa (M. Strathern $1972: 168$ ).

8. Le terme initiation désigne ici des pratiques collectives obligatoires où les initiés sont divisés en classes d'âge et soumis à des épreuves physiques et psychiques qui les font accéder à un nouveau statut. En ce sens, les cultes de célibataires des Huli ou des Enga et les cultes de fertilité des Kuma ou des Melpa n'entrent pas dans cette catégorie. 


\section{B I B L I O G R A P H I E}

BERNDT, R. M.

1962 Excess and Restraint. Chicago, University of Chicago Press.

BIERSACK, A.

1980 The Hidden God. Communication, Cosmology, and Cybernetics among a Melanesian People. $\mathrm{Ph}$. D. dissertation. Ann Arbor, University of Michigan.

1983 «Bound Blood : Paiela 'Conception Theory' Interpreted », Mankind 14 (2) : 85-100.

BUCHBINDER, G. \& R. A. RAPpaport

1976 «Fertility and Death Among the Maring », in P. Brown \& G. BuCHBInDER, eds., Man and Woman in the New Guinea Highlands. Washington, American Anthropological Association : 13-25.

FEII, D. K.

1978 «Enga Women in the Tee Exchange », Mankind 11(3) : 220-230.

1980 «Symmetry and Complementarity: Patterns of Competition and Exchange in the Enga Tee », Oceania $51:$ 20-39.

1984 Ways of Exchange. The Enga Tee of Papua New Guinea. St. Lucia, University of Queensland Press.

1987 The Evolution of Highland Papua New Guinea societies. Cambridge, Cambridge University Press.

Gillison, G.

1980 «Images of Nature in Gimi Thought», in C. Mc CORMACK \& M. Strathern, eds., Nature, Culture and Gender. Cambridge, Cambridge University Press : 143-173.

1983 "Cannibalism Among Women in the Eastern Highlands of Papua New Guinea ", in P. BRown \& D. Tuzin, eds., The Ethnography of Cannibalism. Washington, The Society for Psychological Anthropology : 33-50.

1986 "Le Pénis géant. Le frère de la mère dans les Hautes Terres de Nouvelle-Guinée ", L'Homme $99:$ 41-69.

GLASSE, R. M.

1968 Huli of Papua. A Cognatic Descent System. Paris, EHESS (« Cahiers de l'Homme » VIII).

1974 «Le Masque de la volupté. Symbolisme et antagonisme sexuels sur les hauts plateaux de Nouvelle-Guinée », L'Homme XIV (2) : 79-86.

GODELIER, M.

1982 La Production des Grands Hommes. Pouvoir et domination masculine chez les Baruya de Nouvelle-Guinée. Paris, Fayard («L'Espace du Politique »).

GOLDMAN, L.

1983 Talk Never Dies. The Language of Huli Disputes. London, Tavistock Publications.

GRAY, B.

1973 The Logic of Yandapu Enga Puberty Rites and the Separation of the Sexes: Responses to Ecological and Biological Pressures in New Guinea. Unpublished MA dissertation. University of Sydney.

HeALEY, C. J.

1985 "Pigs, Cassowaries and Gift of the Flesh : A Symbolic Triad in Maring Cosmology », Ethnology, 24 (3) : 153-165.

HERDT, G.

1981 Guardians of the Flute. Idioms of Masculinity. New York, McGraw-Hill.

1982 «Fetish and Fantasy in Sambia Initiation, in G. HeRDr, ed., Rituals of Manhood. Male Initiation in Papua New Guinea. Berkeley, University of California Press : 44-98.

1984a "Semen Transactions in Sambia Culture », in G. HERDT, ed., Ritualized Homosexuality in Melanesia. Berkeley, University of California Press : 167-210. 
1984b «Ritualized Homosexual Behavior in the Male Cults of Melanesia, 1862-1983: An Introduction ", in G. HERDT, ed., Ritualized Homosexuality in Melanesia. Berkeley, University of California Press : 1-81.

1987 The Sambia. Ritual and Gender in New Guinea. New York, Holt, Rinehart \& Winston.

Héritier-Augé, F.

1987 «La Mauvaise odeur l'a saisi », Le Genre humain 15 : 7-17.

Hinton, P. \& G. Mc Call, eds.

1983 Concepts of Conception : Procreation Ideologies in Papua New Guinea, in Mankind 14 (1), special issue.

HogBIN, I.

1943 «A New Guinea Infancy : From Conception to Weaning in Wogeo », Oceania 13 (4) : 285 309.

JORGENSEN, D.

1983 «The Facts of Life, Papua New Guinea Style », Mankind 14 (1) : 1-12.

JUILLERAT, B.

1986 Les Enfants du sang. Société, reproduction et imaginaire en Nouvelle-Guinée. Paris, Éd. de la Maison des Sciences de l'Homme.

KELLY, R. C.

1976 «Witchcraft and Sexual Relations », in P. Brown \& G. BuchBinder, eds., Man and Woman in the New Guinea Highlands. Washington, American Anthropological Association : 36-53.

1977 Etoro Social Structure. A Study in Social Contradiction. Ann Arbor, The University of Michigan Press.

LANDTMAN, G.

1927 The Kiwai Papuans of British New Guinea. London, Macmillan \& Co Ltd.

LEDERMAN R.

1986 What Gifts Engender. Social Relations and Politics in Mendi, Highland Papua New Guinea. Cambridge, Cambridge University Press.

LINDENBAUM, S.

1984 «Variations on a Sociosexual Theme in Melanesia », in G. HerdT, ed., Ritualized Homosexuality in Melanesia. Berkeley, University of California Press : 337-361.

1987 "The Mystification of Female Labors », in J. F. Collier \& S. J. Yanagisako, eds., Gender and Kinship : Toward an Unified Analysis. Stanford, Stanford University Press : 221-243.

LiPUMA, E.

1979 «Sexual Asymmetry and Social Reproduction Among the Maring of Papua New Guinea », Ethnos 44 (1-2) : 34-57.

1981 «Cosmology and Economy Among the Maring of Highland New Guinea », Oceania 51 (4) : 266-285.

1988 The Gift of Kinship. Structure and Practice in Maring Social Organization. Cambridge - New York, Cambridge University Press.

Mandeville, E.

1979 «Sexual Pollution in the New Guinea Highlands », Sociology of Health and Illness 1 (12) : $226-241$.

MEAD, M.

1940 "The Mountain Arapesh », in Anthropological Papers of the American Museum of Natural History 37 (3) : 317-451.

MegGrix, M. J.

1964 "Male-female Relationships in the Highlands of Australian New Guinea », American Anthropologist, special publication, 66 (4) : 204-224.

1965 The Lineage System of the Mae-Enga of New Guinea. Edinburgh - London, Oliver \& Boyd. 
Meigs, A. S.

1976 «Male Pregnancy and the Reduction of Sexual Opposition in a New Guinea Highlands Society », Ethnology 15 (4) : 393-407.

1984 Food, Sex and Pollution : a New Guinea Religion. New Brunswick, NJ, Rutgers University Press.

Mimica, J.

1981 Omalyce. An Ethnography of the Iqwaye View of the Cosmos. Ph. D. dissertation, Canberra, Australian National University.

MoDJeska, N.

1982 «Production and Inequality : Perspectives from Central New Guinea ", in A. J. STRATHERN, ed., Inequality in New Guinea Highlands Societies. Cambridge, Cambridge University Press : 50-108.

MoISSEeff, MariKa

1987 «Entre Maternité et procréation : l'inceste », Patio. Psychanalyse, n.s., $7: 121-145$.

NEWMAN, P. \& D. Boyd

1982 "The Making of Men : Ritual and Meaning in Awa Male Initiation, in G. Herdt, ed., Rituals of Manhood. Male Initiation in Papua New Guinea. Berkeley, University of California Press : 239-285.

Poole, F. J. P.

1982 "The Ritual Forging of Identity : Aspects of Person and Self in Bimin-Kuskusmin Male Initiation ", in G. HERDT, ed., Rituals of Manhood. Male Initiation in Papua New Guinea, Berkeley, University of California Press : 99-154.

1984 «Symbols of Substance: Bimin-Kuskusmin Models of Procreation, Death and Personhood ", Mankind 14 (3) : 191-216.

SCHIEFFELIN, E. L.

1976 The Sorrow of the Lonely and the Burning of the Dancers. New York, St. Martin's Press.

1982 «The Bau a Ceremonial Hunting Lodge : An Alternative to Initiation », in G. HERDr, ed., Rituals of Manhood. Male Initiation in Papua New Guinea, Berkeley, University of California Press : 155-200.

SERPENTI, L. M.

1965 Cultivators in the Swamps. Social Structure and Horticulture in a New Guinea Society (Frederik-Hendrik Island West New Guinea). Assen-Amsterdam, Van Gorgum.

1984 « The Ritual Meaning of Homosexuality and Pedophilia Among the Kimam-Papuans of Irian Jaya », in G. HERDT, ed., Ritualized Homosexuality in Melanesia. Berkeley, University of California Press : 293-317.

SORUM, A.

1984 « Growth and Decay : Bedamini Notions of Sexuality », in G. HERd, ed., Ritualized Homosexuality in Melanesia. Berkeley, University of California Press : 318-336.

STRATHERn, A. J.

1971 The Rope of Moka. Big-Men and Ceremonial Exchange in Mount Hagen, New Guinea. Cambridge, Cambridge University Press.

STRATHERN, M.

1972 Women in Between. Female Roles in a Male World : Mount Hagen, New Guinea. New York, London, Seminar Press.

Strathern, A. J. \& M. Strathern

1969 "Marriage in Melpa », in R. Glasse \& M. Megcrrr, eds., Pigs, Pearshells and Women. Englewood Cliffs, NJ, Prentice Hall : 138-158.

TUZIN, D.

1982 «Ritual Violence Among the llahita Arapesh », in G. Herdr, ed., Rituals of Manhood. Male Initiation in Papua New Guinea. Berkeley, University of California Press : 321-355.

VAN BAAL, J.

1966 Dema : Description and Analysis of Marind-Anim Culture. The Hague, Martinus Nijhoff.

1984 "The Dialectics of Sex in Marind-Anim Culture », in G. HeRDT, ed., Ritualized Homosexuality in Melanesia. Berkeley, University of California Press : 128-166. 
WAGNER, R.

1967 The Curse of Souw. Principles of Daribi Clan Definition and Alliance in New Guinea. Chicago and London, University of Chicago Press.

1977 "Analogic Kinship. A Daribi example ", American Ethnologist 4 (4) : 623-642.

1983 "The Ends of Innocence : Conception and Seduction Among the Daribi of Karimui and the Barok of New Ireland », Mankind 14 (1) : 75-83.

WEINER, A.

1983 La Richesse des femmes ou comment l'esprit vient aux hommes, iles Trobriand. Paris, Le Seuil.

WEINER, J. F.

1982 «Substance, Siblingship and Exchange : Aspects of Social Structure in New Guinea », Social Analysis 11: 3-34.

1987 «Diseases of the Soul : Sickness, Agency and the Men's Cult Among the Foi of New Guinea ", in M. Strathern, ed., Dealing with Inequality. Analysing Gender Relations in Melanesia and Beyond. Cambridge, Cambridge University Press : 255-277.

Williams, F. E.

1936 Papuans of the Trans-Fly. Oxford, The Clarendon Press.

Williamson, M. H.

1983 "Sex Relations and Gender Relations : Understanding Kwoma Conception », Mankind 14 (1) : 13-23.

Young, M. W.

1979 The Ethnography of Malinowski : The Trobriand Islands : 1915-1918. London, Routledge \& Kegan. 


\begin{abstract}
A B S T R A C T
Pascale BonNemère, Reflections about the Representations of Bodily Substances in New Guinea. - Beliefs about bodily substances in some twenty New Guinea societies seem to form a system. After characterizing these substances and underlining their ambivalence, the author draws a parallel between representations linked to conception on the one hand and to ritualized homosexuality in male initiations on the other. It is shown that a woman must be symbolically present so that boys become men. This can be related to the fact that, in the ceremonial exchanges in big men societies, women play an essential mediating role in the transactions which need to be performed successfully in order to become an adult. Finally, the author tries to link modes of marriage and mortuary practices to representations of bodily substances through the principles of reciprocity and substitution.
\end{abstract}

\title{
ZUSA M MENFASS UNG
}

Pascale BONNEMĖkE, Überlegungen $z u$ den Vorstellungen über Körpersubstanzen in Neuguinea. - Die Glaubensvorstellungen über Körpersubstanzen in zwanzig Gesellschaften Neuguineas scheinen systematischen Charakter zu besitzen. Nachdem die Autorin jede dieser Substanzen charakterisiert und ihre Ambivalenz hervorgehoben hat, stellt sie eine Parallele zwischen den Vorstellungen, die sich auf die Empfängnis, und solchen, die sich auf die in den männlichen Initiationen ritualisierte Homosexualität beziehen, her. Sie zeigt in diesem Zusammenhang die Notwendigkeit der symbolischen Präsenz einer Frau auf, damit aus Knaben Männer werden können. Dies wird dann mit der Tatsache in Annäherung gebracht, daß die Frauen in den Transaktionen des Zeremonialtausches der big men-Gesellschaften, dessen erfolgreiche Abwicklung den Übergang zum Erwachsenenstatus sichert, eine wesentliche Vermittlerrolle spielen. Schliesslich versucht die Autorin mittels der Prinzipien Reziprozität und Substitution Heiratstypen und Totenbräuche mit den Vorstellungen über Körpersubstanzen in Verbindung zu bringen.

\section{RESUMEN}

Pascale BONNEMÈRE, Consideraciones relativas a las representaciones de las substancias corporales en Nueva Guinea. - Las creencias relativas a las substancias corporales en una veintena de sociedades de Nueva Guinea parecen formar sistema. Tras haber caracterizado cada una de estas substancias y señalado su ambivalencia, el autor establece un paralelo entre las representaciones relacionadas a la conceptión y las que lo son a la homosexualidad ritualizada en el transcurso de las iniciaciones masculinas. Muestra la necesaria presencia simbólica de una mujer para que los jóvenes se hagan hombres. Esto es entonces relacionado con el hecho de que en los intercambios ceremoniales de las sociedades con big men, las mujeres tengan un papel de mediador esencial en las transaciones cuyo éxito asegura el paso al status de adulto. Para terminar, la autora trata de relacionar tipos de matrimonio y prácticas mortuorias a las concepciones sobre las substancias corporales por medio de los principios de reciprocidad y substitución. 